\title{
Mediating the relationship between loneliness and cognitive function: the role of depressive and anxiety symptoms
}

\author{
Joanna McHugh Power, Jianjun Tang, Rose Ann Kenny, Brian A Lawlor \& \\ Frank Kee
}

To cite this article: Joanna McHugh Power, Jianjun Tang, Rose Ann Kenny, Brian A Lawlor \& Frank Kee (2020) Mediating the relationship between loneliness and cognitive function: the role of depressive and anxiety symptoms, Aging \& Mental Health, 24:7, 1071-1078, DOI: 10.1080/13607863.2019.1599816

To link to this article: https://doi.org/10.1080/13607863.2019.1599816

\section{Published online: 07 Apr 2019.}

\section{Submit your article to this journal $\widetilde{3}$}

Џlll Article views: 569

Q View related articles $\sqsubset$

View Crossmark data $₫$

Citing articles: 1 View citing articles $₫$ 


\title{
Mediating the relationship between loneliness and cognitive function: the role of depressive and anxiety symptoms
}

\author{
Joanna McHugh Power ${ }^{\mathrm{a}, \mathrm{b}, \mathrm{c}}\left(\mathbb{D}\right.$, Jianjun Tang ${ }^{\mathrm{d}}$ (D), Rose Ann Kenny ${ }^{\mathrm{c}}$, Brian A Lawlor ${ }^{\mathrm{c}}$ and Frank Kee ${ }^{\mathrm{b}}$ \\ ${ }^{a}$ School of Business National College of Ireland, Dublin 1, Ireland; 'bK CRC Centre of Excellence for Public Health Queen's University \\ Belfast, Belfast, United Kingdom of Great Britain and Northern Ireland; ' Institute of Neuroscience \& School of Medicine, Trinity College, \\ Dublin 2, Ireland; ${ }^{\mathrm{d} S c h o o l}$ of Agricultural Economics and Rural Development, Renmin University of China, Beijing, China
}

\begin{abstract}
Objective: To evaluate the relationship between loneliness and cognitive functioning, and whether depressive and anxiety symptoms have intermediate roles therein.

Methods: Information about 7,433 participants of the Irish Longitudinal Study on Ageing (a prospective, representative cohort study), aged over 50, was collected at three time-points two years apart, and analysed using Structural Equation Modelling to assess whether depressive and anxiety symptoms mediate the relationship between loneliness and cognitive functioning. Cognitive functioning was measured as a latent factor, with four indicators: measures of immediate and delayed word recall, verbal fluency, and a global measure (the MMSE). Loneliness was measured using the UCLA Loneliness scale, depressive symptoms using the CES-D-ML scale, and anxiety symptoms using the HADS-A scale.

Results: Loneliness at time-point 1 predicted cognitive functioning at time-point $3, \beta=-0.103$, $p<0.001$, and depressive $(\beta=0.426, p<0.001)$ and anxiety $(\beta=0.410, p<0.001)$ symptoms at time-point 2 . Depressive $(\beta=-0.020, p=0.001)$ but not anxiety $(\beta=-0.000, p=0.658)$ symptoms mediated the relationship between loneliness and cognitive functioning, total effect: $\beta=$ $-0.123, p<0.001$.

Conclusion: The relationship between loneliness and cognitive functioning is in part explained by its relationship with depressive symptoms. Statistically, the mediation model helps us understand possible mechanisms through which loneliness impacts cognitive functioning. Results have implications for cognitive functioning interventions for older adults, and imply that loneliness is also a worthwhile target for intervention.
\end{abstract}

\section{ARTICLE HISTORY}

Received 23 October 2018

Accepted 19 March 2019

\section{KEYWORDS}

Ageing; cognitive function; mental health; structural equation modelling

\section{Introduction}

Evidence is accumulating for the existence of a relationship between loneliness and cognitive outcomes. Loneliness is related to higher risk of cognitive decline (Boss, Kang, \& Branson, 2015) and to the development of Alzheimer's Disease (Wilson et al., 2007). Loneliness is most commonly defined as a perceived discrepancy between actual and desired levels of social contact with others (Peplau \& Perlman, 1982) or in the quality of these contacts (Hawkley et al., 2008). In a comprehensive discussion of the manner through which loneliness might impact cognitive functioning, Hawkley and Cacioppo suggest some pathways (Cacioppo \& Hawkley, 2009) that may explain this effect. Such pathways were thought to include: elevated Hypothalamic-Pituitary-Adrenal (HPA) axis activation/neuroinflammation; age-related reductions in social stimulation; cognitive load as a result of the hypervigilance associated with loneliness (they hypothesise that lonely individuals are hyper-vigilant to social threats in the environment); depression and sedentary behaviour; reduced social interaction (since loneliness and cognitive decline may both be caused by reduced social interaction); and diminished sleep quality (since loneliness is known to have an impact on the quality of sleep, which can in turn adversely impact cognitive functioning).
As well as these suggested mechanisms, there is cause to expect mediating roles of depressive and anxiety symptoms specifically in the relationship between loneliness and cognitive functioning. Firstly, loneliness represents a risk factor for depression (Cacioppo, Hawkley, \& Thisted, 2010; Heikkinen \& Kauppinen, 2004; Luanaigh \& Lawlor, 2008) and is also associated with anxiety (Beutel et al., 2017; Muyan et al., 2016). In addition, the links between depressive and anxiety symptoms and cognitive functioning (thought to be causal), are well documented. Higher levels of anxiety have previously been shown to relate to poorer cognitive function in older adults (Wetherell, Reynolds, Gatz, \& Pedersen, 2002) alongside worse decline in processing speed over a six year period (Petkus, Reynolds, Wetherell, Kremen, \& Gatz, 2017). Higher levels of depressive symptoms are also known to be related to lower levels of cognitive performance (Weisenbach, Boore, \& Kales, 2012; Yaffe et al., 1999). Depression was also previously found to mediate the association between receipt of social support and cognitive function in an ageing population (Gow, Corley, Starr, \& Deary, 2013). Given that depressive and anxiety symptoms represent the most common mental disorders of later life (Byers, Yaffe, Covinsky, Friedman, \& Bruce, 2010) it would appear important to explore their contribution to negative outcomes such as poor cognitive 
functioning, in the context of loneliness. Loneliness may constitute a form of stress with deleterious effects on psychological, physical, and cognitive health (Cacioppo, Grippo, London, Goossens, \& Cacioppo, 2015) and since it has a peak during later life (Qualter et al., 2015) it is vital to evaluate its impact on these outcomes.

However, the field is lacking empirical evidence on the potential mediating role of depression and anxiety in the relationship between loneliness and cognitive functioning. Using data from the Irish Longitudinal Study on Ageing, we investigated whether depressive and anxiety symptoms constitute mediators of the purported relationship between loneliness and cognitive functioning, among adults aged over 50.

\section{Hypothesis}

Depressive and anxiety symptoms constitute mediators of the (negative) association between loneliness and cognitive functioning.

\section{Research design and methods}

TILDA (Irish Longitudinal Study on Ageing) is a representative, longitudinal cohort study of ageing among adults in Ireland. Participants (8504 in total) over the age of 50 were recruited via stratified random sampling based on all geographical units in the country. Informed consent was obtained from all individual participants included in the study. More information on the design of the TILDA study is available elsewhere (Kenny et al., 2010). The baseline assessments were collected between 2009 and 2011. Follow-up assessments have since been conducted every two years. The current analysis utilises data collected during the baseline (wave one), and waves two and three of the study.

\section{Participants}

Of the 8504 TILDA participants, 8163 were over the age of 50 , and as such included in the current analyses. The remaining participants were spouses or partners of the participants initially contacted, who were also invited to participate in the study regardless of age. The mean age of participants at baseline was 63.83 (age range of 50-105; standard deviation $=9.78$ ) and $45.8 \%$ were male. Each participant completed a home-based interview with an assessor, a paper-based questionnaire in their own time, and were invited to participate in a health assessment in a TILDA health centre if possible. At wave two, 1180 participants had been lost to follow-up (205 had died, 166 were untraceable, and 809 refused). By wave three, a further 304 were deceased. Between baseline and wave three, a total of 1089 participants were refused or withdrawn, 89 were untraceable, and 56 were lost to follow-up for other reasons.

Participants were asked at baseline (wave 1) whether they had been told by a doctor that they had any of the following: Alzheimer's disease, dementia, organic brain syndrome, senility, a serious memory impairment, or any emotional, nervous, or psychiatric problems such as depression and anxiety. Eight individuals had been told they had

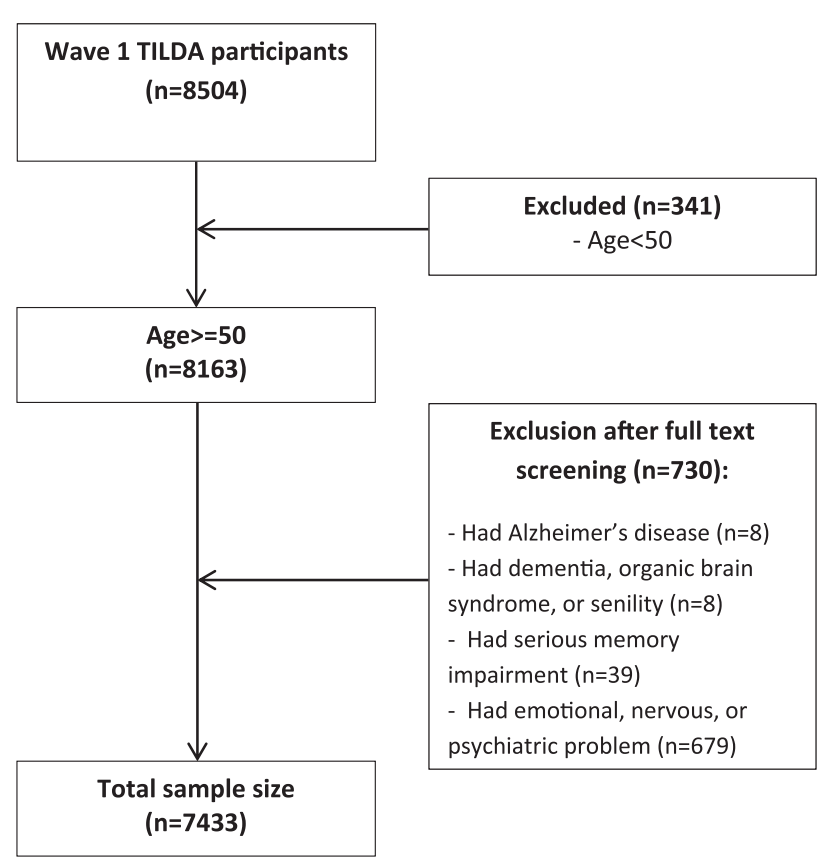

Figure 1. Flow diagram showing the sample selection process.

Alzheimer's disease; eight had been told they had dementia, organic brain syndrome, or senility; 39 had been told that they had a serious memory impairment, and 679 had been told that they had an emotional, nervous, or psychiatric problem such as depression or anxiety. These groups amounted to 730 individuals in total. Data pertaining to these 730 individuals were excluded from further analysis, yielding a final sample size of 7433 . Figure 1 displays a flow diagram showing the sample selection process.

All procedures performed in studies involving human participants were in accordance with the ethical standards of the Faculty of Health Sciences Research Ethics Committee at Trinity College Dublin and with the 1964 Helsinki declaration (and its later amendments, and comparable ethical standards).

\section{Measures}

Covariates included in the model were age, sex, education level (categorised as having received no education, or less than second level, having received second level education, or having received tertiary level education or higher), and physical health (measured using two self-reported items: number of cardiovascular conditions, including angina, heart attack, heart failure, stroke, and abnormal heart rhythm etc.; and number of chronic conditions, including the above cardiovascular conditions, hypertension, diabetes, asthma, bronchitis, cancer, arthritis etc.).

\section{Cognitive measures}

Outcomes of interest were cognitive in nature and measured episodic memory, global cognitive functioning, and executive functioning. The cognitive measures deployed in TILDA were chosen according to specific guidelines set out by the Health and Retirement Study (HRS), who were the first study to attempt cognitive evaluation of participants in a national survey. Following these guidelines, measures were chosen to be sensitive to change over time, 
administrable in a survey context, to provide data that is comparable to other national surveys, and to be valid and reliable (Kenny et al., 2010).

Participants engaged in an immediate word list recall task where they heard a list of ten nouns read to them by a recorded voice and had to repeat as many as they could. This task was adopted directly from the HRS, and the list of ten words were the same as those used in the HRS. Delayed word list recall was evaluated by repeating this task following a distractor task. More information about the development of the immediate and delayed word list recall tasks, which were developed specifically for the HRS, are available elsewhere (Ofstedal, Fisher \& Herzog, 2005).

The Mini Mental State Examination (MMSE) was used as a more global measure of cognitive functioning in both TILDA and the HRS (Folstein, Folstein, \& McHugh, 1975). The MMSE is the best known and most frequently used brief method of evaluating cognitive impairment in older adults (Arevalo-Rodriguez et al., 2015), and is used both in research and clinical settings. The MMSE evaluates attention, orientation, memory, registration, recall, calculation, language, and praxis.

Verbal fluency was measured using an animal naming task whereby the participant was instructed to name as many animals as they could in 60 seconds. The task is originally derived from Thurstone's Word Fluency Test and is used in the HRS and ELSA. The animal naming task is also considered to be a measure of executive functioning (Fisk \& Sharp, 2004) although this task may not be the ideal measure of executive functioning (Shao, Janse, Visser, \& Meyer, 2014).

\section{Psychosocial measures}

Loneliness was measured using a five-item version of the UCLA loneliness scale (Russell, 1996). Because of poor factor loading, item 4 ("Do you feel in tune with the people around you?") was dropped from the models reported below. Depressive symptoms were measured using the validated and reliable 20-item Centre for Epidemiological Studies (CES-D) scale (Radloff, 1977). Because of poor factor loading, item 4 ("I felt that I was just as good as other people"), 15 ("People were unfriendly"), and 19 ("I felt that people disliked $\mathrm{me}^{\prime \prime}$ ) were dropped from the models reported below. Qualitatively, these items could be interpreted as describing a subcategory of depressive symptoms concerning social success, which may be better described as a separate construct. Anxiety symptoms were measured using the Hospital Anxiety \& Depression Scale Anxiety (HADS-A) Subscale (Zigmond \& Snaith, 1983). In this scale, all items loaded well onto the Anxiety latent variable.

\section{Data analysis}

All analyses were conducted in $\mathrm{R}$ software (version 3.1.0). Structural Equation Modelling (SEM) was used to evaluate whether depressive and anxiety symptom measures mediated the putative relationships between loneliness and cognitive outcomes. Cognitive measures may not individually represent cognitive function perfectly, despite each measure having some degree of explanatory power. Taking the four cognitive outcomes as separate observed dependent variables would increase measurement error. Thus we decided to further explore our data by characterising cognitive functioning as a latent variable measured by five items: Immediate Recall (two separate trials thereof, with error variances allowed to co-vary between the two and with Delayed Recall), Delayed Recall, MMSE, and Verbal Fluency. Loneliness was also treated as a latent variable with 4 items in the UCLA loneliness scale as indicators. Depression was treated similarly as a latent variable composed of 16 items of the CES-D-ML scale with the item about loneliness removed to avoid multicollinearity (Cacioppo, Hughes, Waite, Hawkley, \& Thisted, 2006) and Anxiety was also treated as a latent variable composed of all 7 items in the HADS-A scale. Omega values were computed as reliability coefficients with a cut off of 0.7 taken as demonstrating acceptable reliability (Revelle \& Zinbarg, 2009).

As well as facilitating the inclusion of latent factors like Cognitive Function in the current example, SEM is useful when assessing mediation since it caters for endogeneity between cause and effect, while estimating several equations simultaneously (Gunzler, Chen, Wu, \& Zhang, 2013). We used the 'lavaan' package (version 0.5-20) in $\mathrm{R}$ to conduct the mediation analysis (Rosseel, 2012). Another feature of this package is that it facilitates multiple imputation for missingness, here using the full information maximum likelihood (FIML) approach. The lavaan package outputs several measures of goodness of fit including the Comparative Fit Index (CFI), Tucker Lewis Index (TLI), Root Mean Square Error Approximation (RMSEA) and the Standardised Root Mean Square Residual (SRMR). The lavaan package also describes standardised beta coefficients for all specified natural direct and total pathways in the model as well as estimates of the natural indirect effects (i.e those whose effects are via each specified mediator). The natural direct effect here refers to the difference in risk of cognitive decline between the individuals who are lonely and those who are not lonely, conditional on a given level of the mediators (depressive and anxiety symptoms). The natural indirect effect, meanwhile, refers to the difference in risk of cognitive decline between the individuals who are lonely and those who are not lonely with and without symptoms of depression and anxiety.

\section{Results \\ Sample characteristics}

The characteristics of 7,433 participants in the current analysis are described below in Table 1. All participants were aged over 50 , with a mean age of 63.99 , and $46.64 \%$ were male, with almost one third of participants (30.7\%) without any formal educational qualifications. Performance in immediate and delayed recall was similar to that previously reported in the HRS, a nationally representative study of U.S. adults aged over 50 (where average scores of 6.06 in immediate and 5.21 in delayed word recall were recorded; Runge, Craig \& Jim, 2015). Scores on the MMSE indicated that participants overall had good cognitive functioning, and scores on the verbal fluency task were superior to norms in the population aged over 50 previously reported which ranged from 20 in the 50-59 age group to 13 in the 90-95 age group (Tombaugh, Kozak, \& Rees, 1999). The 
Table 1. Sample characteristics of 7433 participants at baseline (wave one) of the TILDA study.

\begin{tabular}{lcc}
\hline Variable name & Mean/Percentage & SD \\
\hline Age & 63.99 & 9.83 \\
Gender & & \\
Male & $46.64 \%(n=3467)$ & \\
Female & $53.36 \%(n=3966)$ & \\
Education Level & & \\
No Education & $30.7 \%(n=2282)$ & \\
Secondary Education & $40.2 \%(n=2986)$ & \\
Third level or Higher Education & $29.1 \%(n=2161)$ & \\
Immediate Recall (Trial 1) & 5.69 & 1.74 \\
Immediate Recall (Trial 2) & 7.44 & 1.89 \\
Delayed Recall & 5.87 & 2.35 \\
MMSE & 28.31 & 2.17 \\
Verbal Fluency & 20.3 & 7.07 \\
Number of Cardiovascular Conditions & 0.19 median $=0$ & 0.52 \\
Number of Chronic Illnesses Reported & 1.67 median $=1$ & 1.42 \\
Loneliness & 1.81 & 2.08 \\
Depressive Symptoms & 4.79 & 6.22 \\
Anxiety Symptoms & 3.14 & 3.12 \\
\hline
\end{tabular}

sample were generally healthy with a median of 0 cardiovascular and 1 chronic illnesses reported. Scores on loneliness were slightly lower, on average, for the current subsample of TILDA than for the entire sample (mean $=2$, Barrett et al., 2011). Scores of depressive symptoms were not comparable to the TILDA sample since the item on loneliness was excluded.

\section{Mediation within linear SEM}

A linear SEM model with mediation pathways was created to evaluate whether depressive and anxiety symptoms represent mechanisms through which loneliness impact Cognitive Function among older adults. The model converged after 85 iterations. The model fit indices indicated a mediocre to acceptable fit, $\chi_{577}^{2}=6194, p<.001$; but CFI $=0.89, \mathrm{TLI}=0.88, \mathrm{RMSEA}=0.046\left(\mathrm{Cl}_{90}=0.044,0.047\right)$, SRMR $=0.083$. The fit indices RMSEA and SRMR here are acceptable (Hu \& Bentler, 1999) but the CFI and TLI are just below cut off. The measurement component of the model is detailed in Table 2. Cognitive Functioning as a latent variable had generally high factor loadings (between 0.578 and 0.753 ) with an omega value of 0.70 which appears to indicate reliability. Loneliness as a latent variable had generally acceptable factor loadings (0.694-0.771) with an acceptable omega value of 0.83 . Factor loadings for depressive symptoms were between 0.403 and 0.721 , with an omega value of 0.86 . Finally, anxiety symptoms had a high omega value (0.78) and factor loadings were between 0.484 and 0.665 .

Regression paths showed that Loneliness at wave 1 had a small association with Cognitive Function at wave $3, \beta=$ $-0.103, p<.001$, while depressive symptoms at wave 2 also had a very small association with Cognitive Function, $\beta=-0.047, p=.004$, but not anxiety symptoms, $\beta=$ $-0.001, p=.958$. Depressive $(\beta=0.426, p<.001)$ and anxiety symptoms $(\beta=0.410, p<.001)$ were both predicted by Loneliness, with a moderate effect size (see Figure 2 ). The mediation analysis showed that an indirect pathway exists between Loneliness and Cognitive functioning via depressive symptoms $(\beta=-0.020, p=.005)$ but not via anxiety symptoms ( $\beta=-0.000, p=.958)$, with a small total effect ( $\beta$ $=-0.123, p<.001$; see Table 3 ). For each increase of one standard deviation in loneliness, there is 0.103 of a standard deviation decrease in cognitive function latent scores through the direct effect. Subsequently, the size of the indirect effects via Depressive symptoms was $19.4 \%$ $(-0.020 /-0.103)$ of that direct effect.

\section{Discussion}

We hypothesised that both depressive and anxiety symptoms would mediate the relationship between loneliness and cognitive functioning outcomes. We found that depressive symptoms partly mediated the relationship between loneliness and cognitive functioning outcomes, but anxiety symptoms did not. Loneliness has previously been identified as a risk factor for depressive symptom onset (Cacioppo et al., 2010), and has been shown to be associated with anxiety symptoms (Beutel et al., 2017; Muyan et al., 2016) and both of these findings were replicated in the current study. We also replicated previous findings that show an effect of depressive symptoms on cognitive functioning (Cacioppo et al., 2010; Heikkinen \& Kauppinen, 2004; Luanaigh \& Lawlor, 2008). Previously, mechanisms through which loneliness may impact cognitive function were outlined as avenues for future research (Cacioppo \& Hawkley, 2009). The current findings support the existence of one such suggested mechanism, namely, depressive symptoms. We found no evidence that anxiety mediated the relationship between loneliness and cognitive decline, and we note that others (de Bruijn et al., 2014) have even reported a beneficial impact of anxiety on performance of some cognitive measures (Potvin et al., 2013).

The indirect effect of loneliness on cognitive functioning via depressive symptoms was small relative to the direct effect. This suggests that loneliness exerts the majority of its effect on cognitive functioning either directly, or through mechanisms not accounted for in the current analysis. Future research could also investigate multiple mediators in tandem to explore the proportion of the total effect between loneliness and cognitive function that is accounted for by other variables. These other variables could include those suggested by Cacioppo and Hawkley (2009) which include inflammation, HPA axis dysfunction, cognitive load, and sedentary behaviour. Adolphs has suggested that all cognition is social in nature (Adolphs, 2003) and thus, if loneliness in part reflects an individual's social functioning, a correlation between the two variables should be expected. Additionally, we must allow for the possibility that loneliness itself may be an umbrella term for different facets of social functioning such as romantic loneliness, social loneliness, and emotional loneliness (Fokkema, de Jong Gierveld, \& Dykstra, 2012; Sønderby \& Wagoner, 2013), and that these subtypes of loneliness may impact cognitive functioning via different mechanisms.

The current analysis utilised data from a large representative study of older people, which is a methodological strength since sampling bias is minimised and results are likely to generalisable to the older Irish population. In addition, three waves of data were used in the analysis, which is required to fully investigate a potential mediation effect (Preacher, 2015). However, only two years passed between each wave of data collection, meaning that the results are limited in the extent to which they can be used to 
Table 2. Measurement Model for loneliness, cognitive function, depressive symptoms, and anxiety symptoms as latent variables in waves 1 , 2 , and 3 of TILDA.

\begin{tabular}{|c|c|c|c|c|}
\hline Item & Factor Loading & $p$ value & $R^{2}$ & Reliability (omega) \\
\hline Cognitive Function & & & & 0.70 \\
\hline Immediate Recall (Trial 1) & 0.697 & $<.001$ & 0.486 & \\
\hline Immediate Recall (Trial 2) & 0.753 & $<.001$ & 0.567 & \\
\hline Delayed Recall & 0.746 & $<.001$ & 0.556 & \\
\hline MMSE & 0.636 & $<.001$ & 0.404 & \\
\hline Verbal Fluency & 0.578 & $<.001$ & 0.334 & \\
\hline Loneliness & & & & 0.83 \\
\hline Item 1 ("How often do you feel that you lack companionship?") & 0.694 & $<.001$ & 0.482 & \\
\hline Item 2 ("How often do you feel left out?") & 0.771 & $<.001$ & 0.595 & \\
\hline Item 3 ("How often do you feel isolated from others?") & 0.770 & $<.001$ & 0.593 & \\
\hline Item 5 ("How often do you feel lonely?") & 0.760 & $<.001$ & 0.578 & \\
\hline Depressive Symptoms & & & & 0.86 \\
\hline Item 1 ("I was bothered by things that usually don't bother me") & 0.499 & $<.001$ & 0.249 & \\
\hline Item 2 ("I did not feel like eating") & 0.403 & $<.001$ & 0.162 & \\
\hline Item 3 ("I felt that I could not shake off the blues") & 0.633 & $<.001$ & 0.401 & \\
\hline Item 5 ("I had trouble keeping my mind on what I was doing") & 0.507 & $<.001$ & 0.257 & \\
\hline Item 6 ("I felt depressed") & 0.721 & $<.001$ & 0.519 & \\
\hline Item 7 ("I felt that everything I did was an effort") & 0.644 & $<.001$ & 0.415 & \\
\hline Item 8 ("I felt hopeful about the future") & 0.432 & $<.001$ & 0.186 & \\
\hline Item 9 ("I thought my life had been a failure") & 0.428 & $<.001$ & 0.183 & \\
\hline Item 10 ("I felt fearful") & 0.566 & $<.001$ & 0.321 & \\
\hline Item 11 ("My sleep was restless") & 0.433 & $<.001$ & 0.188 & \\
\hline Item 12 ("I was happy") & 0.547 & $<.001$ & 0.300 & \\
\hline Item 13 ("I talked less than usual") & 0.468 & $<.001$ & 0.219 & \\
\hline Item 16 ("I enjoyed life") & 0.542 & $<.001$ & 0.294 & \\
\hline Item 17 ("I had crying spells") & 0.488 & $<.001$ & 0.238 & \\
\hline Item 18 ("I felt sad") & 0.662 & $<.001$ & 0.439 & \\
\hline Item 20 ("I could not get going") & 0.619 & $<.001$ & 0.383 & \\
\hline Anxiety Symptoms & & & & 0.78 \\
\hline Item 1 ("I feel tense or wound up") & 0.665 & $<.001$ & 0.442 & \\
\hline Item 2 ("I get a sort of frightened feeling as if something awful is about to happen") & 0.630 & $<.001$ & 0.397 & \\
\hline Item 3 ("Worrying thoughts go through my mind") & 0.657 & $<.001$ & 0.432 & \\
\hline Item 4 ("I can sit at ease and feel relaxed") & 0.539 & $<.001$ & 0.290 & \\
\hline Item 5 ("I get a sort of frightened feeling like butterflies in the stomach") & 0.484 & $<.001$ & 0.234 & \\
\hline Item 6 ("I feel restless as if I have to be on the move") & 0.529 & $<.001$ & 0.280 & \\
\hline Item 7 ("I get sudden feelings of panic") & 0.640 & $<.001$ & 0.410 & \\
\hline
\end{tabular}

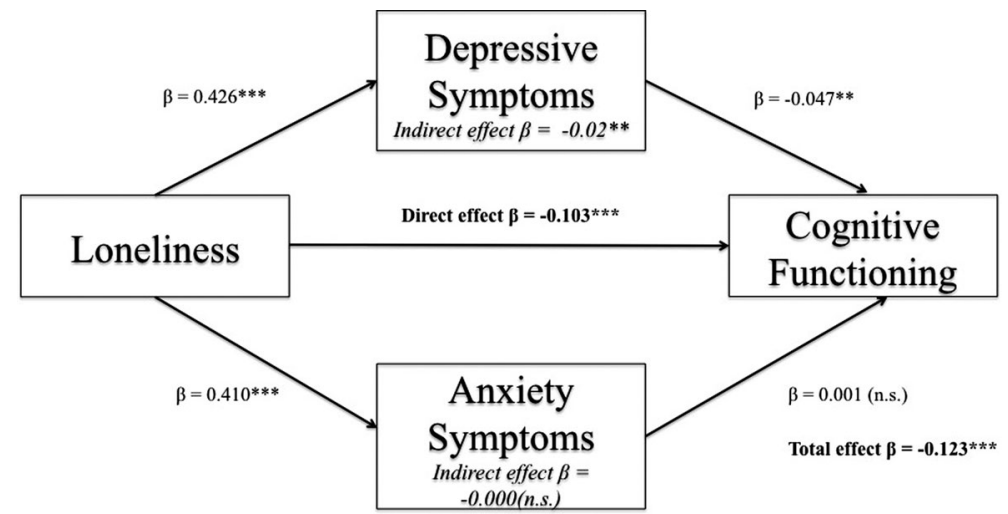

Figure 2. Mediation model showing the relationship between Loneliness and Cognitive Functioning as mediated by both depressive and anxiety symptoms scores. Anxiety symptoms were not a mediator ( ${ }^{* * *}=p<.001 ; * *=p<.01{ }^{*}=p<.05 ; \mathrm{n} . \mathrm{s}$. $\left.=p>.05\right)$.

generalise about longer term associations between loneliness and cognitive functioning. It is possible that the impacts of loneliness and depressive and anxiety symptoms on cognitive functioning may take longer to manifest. To further examine this question, more waves of longitudinal data are required.

We used a structural equation modelling approach which has several advantages over a regression-based mediation approach, including the ability to simultaneously model pathways and reduce type 1 error due to multiple comparisons; the flexibility to specify variables as both predictors and outcomes; and the option to specify variables as latent factors to reduce measurement error. We advocate the use of this approach, since the majority of current psychological mediation analysis is conducted using a regression-based method (Preacher, 2015).
Methodological weaknesses are also inherent in the current analysis. In relation to measurement, we used performance on an animal naming task as an indicator of executive functioning, though we acknowledge that this is not the ideal measure (Shao et al., 2014). Future research investigating the impact of loneliness on executive functioning could measure this domain more comprehensively. This is particularly pertinent since executive functioning is affected in individuals with late-life depression (Alexopoulos, 2003). Thus, future studies with more detailed evaluations of executive functioning should reevaluate the potential contributions of loneliness and depression. Additionally, a more detailed and appropriate measurement of executive function may yield a different pattern of results in relation to anxiety as a mediator, since anxiety has been found to be associated with diminished 
Table 3. SEM Mediation Results for Cognitive Functioning at wave 3 as an outcome, Loneliness at Wave 1 as the independent variable, and with a) Depressive Symptoms, and b) Anxiety Symptoms, both at wave 2, as mediators.

\begin{tabular}{lcrrr}
\hline Regression Path & Standardised beta coefficients & Standard error & $Z$ & $p$ value \\
\hline Pathways from Loneliness & & & & \\
Loneliness -> Cognitive Functioning & -0.103 & 0.025 & -5.512 & $<.001$ \\
Loneliness -> Depressive Symptoms & 0.426 & 0.020 & 24.071 & $<.001$ \\
Loneliness -> Anxiety Symptoms & 0.410 & 0.020 & 22.096 & $<.001$ \\
Pathways to Cognitive Functioning & & & & \\
Depressive Symptoms & -0.047 & 0.020 & -2.845 & .004 \\
Anxiety Symptoms & -0.001 & 0.021 & -0.053 & .958 \\
Age & -0.473 & 0.002 & -28.753 & $<.001$ \\
CVD Conditions & -0.022 & 0.039 & -1.573 & .116 \\
Sex & -0.127 & 0.037 & -9.181 & $<.001$ \\
Education & 0.331 & 0.027 & 21.872 & $<.001$ \\
Mediating Pathways & & & & \\
Indirect Effect via Depressive Symptoms & -0.020 & 0.009 & -2.839 & .005 \\
Indirect Effect via Anxiety Symptoms & -0.000 & 0.009 & -0.053 & .958 \\
Total Effect & -0.123 & 0.020 & -8.257 & $<.001$ \\
\hline
\end{tabular}

executive function in later life (Beaudreau \& O'Hara, 2009; Yochim, Mueller \& Segal, 2013). Next, the RMSEA and CFI yielded inconsistent model-fit evaluations but we note that RMSEA is better suited to confirmatory and large sample situations like ours (Rigdon, 1996) and that CFI may be low when some covariates (e.g. age, education, physical health etc.) are less correlated with the other observed variables and among themselves (Lai \& Green, 2016). Finally, the analysis was based on the surveyed rather than samplingweighted cohort, causing some uncertainty surrounding the effect estimates compared to the population-representative estimates.

Our sample had a very broad age range spanning more than 50 years, which may mean that results lack the granularity to provide meaningful comment on the nature of loneliness and its consequences in different age groups. Future research could explore the moderating role of age on the association between loneliness and cognitive functioning - our current analysis controlled for age but did not consider it as a moderating factor.

We evaluated mediation between loneliness and cognitive outcomes, but we do not rule out the possibility that mediation is also germane to the relationships between cognitive function and other social factors. It is possible that depressive symptoms also mediate the putative relationships between these other social factors and cognitive functioning, since an overall significant effect between predictors is not necessary for mediation to occur (MacKinnon \& Fairchild, 2009). Other possible hypotheses and explanations have not been formally tested here but should be considered in future research. For example, some executive and cognitive functions and behaviours, may be directly changed by socioeconomic conditions, through stress mechanisms, which are also likely to affect mood and mental wellbeing (Bickel, Moody, Quisenberry, Ramey, \& Sheffer, 2014).

We must also be aware of the possibility that individuals with reduced cognitive function may be less likely to engage socially as a result, notably those who may be in a prodromal state of dementia (Saczynski et al., 2006). This possibility would potentially be reflected in data that showed that cognitive functioning predicted loneliness, rather than vice versa, and that mechanisms involving depression and anxiety would play a possible role in this relationship. We conducted additional analysis to explore this possibility and found that while cognitive functioning at wave 1 did predict loneliness at wave 3, cognitive functioning predicted neither depression nor anxiety at wave 2 and there was no evidence of any mediation. Taken with the main findings presented above, we can conclude that there likely exist reciprocal effects between loneliness and cognitive functioning (as has been found previously with memory specifically (Ayalon, Shiovitz-Ezra, \& Roziner, 2016)) but that depression and anxiety do not operate as mediators in both directions of the relationship.

Results have implications for the wellbeing and health of older adults. We can conclude that efforts to reduce loneliness may to some extent foster better cognitive function at follow-up, although randomised controlled trials are needed to demonstrate this conclusively. This finding may be particularly important for older adults who for health reasons cannot engage in physical activity but wish to have their lifestyle contribute positively towards their cognitive health. This would be particularly advised for older adults who have experienced symptoms of depression, since according to our model, part of the effect exerted by loneliness on cognitive function is via depressive symptoms. Policy should reflect these findings for there is a growing body of evidence that social factors constitute a health "behaviour" that protects against important clinical outcomes in later life. As such, reducing loneliness and engaging in social activities may in itself represent a health intervention for older adults.

\section{Acknowledgments}

We wish to thank the staff and participants of TILDA for their role in this study. The project has been designed and implemented by the TILDA Study Team (C) Department of Health and Children. Copyright and all other intellectual property rights relating to the data are vested in TILDA.

\section{Disclosure statement}

No potential conflict of interest was reported by the authors.

\section{Funding}

Funding was received by Joanna McHugh Power in the form of a Leadership in Ageing Research Fellowship grant (from the Centre of Ageing Research and Development in Ireland, now the Institute of Public Health). The TILDA data have been co-funded by the Government of Ireland through the Office of the Minister for Health 
and Children, by Atlantic Philanthropies, and by Irish Life; have been collected under the Statistics Act, 1993, of the Central Statistics Office.

\section{ORCID}

Joanna McHugh Power (D) http://orcid.org/0000-0002-7387-3107

Jianjun Tang (D) http://orcid.org/0000-0002-6994-2093

\section{References}

Adolphs, R. (2003). Cognitive neuroscience of human social behaviour. Nature Reviews Neuroscience, 4(3), 165-178.

Alexopoulos, G. S. (2003). Role of executive function in late-life depression. The Journal of Clinical Psychiatry, 64, 18-23.

Arevalo-Rodriguez, I., Smailagic, N., Roque I Figuls, M., Ciapponi, A., Sanchez-Perez, E., Giannakou, A., ... Cullum, S. (2015). Mini-Mental State Examination (MMSE) for the detection of Alzheimer's disease and other dementias in people with mild cognitive impairment (MCI). Cochrane Database of Systematic Reviews, 3, CD010783.

Ayalon, L., Shiovitz-Ezra, S., \& Roziner, I. (2016). A cross-lagged model of the reciprocal associations of loneliness and memory functioning Psychology and Aging, 31(3), 255-261.

Barrett, A., Burke, H., Cronin, H., Hickey, A., Kamiya, Y., Kenny, R.A., ... \& Whelan, B. (2011). Fifty plus in Ireland 2011: First results from The Irish Longitudinal Study on Ageing (TILDA). Dublin: Trinity College Dublin.

Beaudreau, S. A., \& O'Hara, R. (2009). The association of anxiety and depressive symptoms with cognitive performance in communitydwelling older adults. Psychology and Aging, 24(2), 507-512.

Beutel, M. E., Klein, E. M., Brähler, E., Reiner, I., Jünger, C., Michal, M., ...., Lackner, K. J. (2017). Loneliness in the general population: Prevalence, determinants and relations to mental health. $B M C$ Psychiatry, 17(1), 97.

Bickel, W. K., Moody, L., Quisenberry, A. J., Ramey, C. T., \& Sheffer, C. E. (2014). A competing neurobehavioral decision systems model of SES-related health and behavioural disparities. Preventive Medicine, $68,37-43$

Boss, L., Kang, D. H., \& Branson, S. (2015). Loneliness and cognitive function in the older adult: A systematic review. International Psychogeriatrics, 27(04), 541-553.

Byers, A. L., Yaffe, K., Covinsky, K. E., Friedman, M. B., \& Bruce, M. L. (2010). High occurrence of mood and anxiety disorders among older adults: The National Comorbidity Survey Replication. Archives of General Psychiatry, 67(5), 489-496.

Cacioppo, J. T., \& Hawkley, L. C. (2009). Perceived social isolation and cognition. Trends in Cognitive Sciences, 13(10), 447-454.

Cacioppo, J. T., Hawkley, L. C., \& Thisted, R. A. (2010). Perceived social isolation makes me sad: 5 year cross-lagged analysis of loneliness and depressive symptomatology in the CHASRS Study. Psychology \& Aging, 25(2), 453-463.

Cacioppo, J. T., Hughes, M. E., Waite, L. J., Hawkley, L. C., \& Thisted, R. A. (2006). Loneliness as a specific risk factor for depressive symptoms: Cross-sectional and longitudinal analyses. Psychology \& Aging, 21(1), 140-151.

Cacioppo, S., Grippo, A. J., London, S., Goossens, L., \& Cacioppo, J. T. (2015). Loneliness: Clinical import and interventions. Perspectives on Psychological Science, 10(2), 238-249.

de Bruijn, R. F. A. G., Direk, N., Mirza, S. S., Hofman, A., Koudstaal, P. J., Tiemeier, H., \& Ikram, M. A. (2014). Anxiety is not associated with the risk of dementia or cognitive decline: The Rotterdam Study. The American Journal of Geriatric Psychiatry, 22(12), 1382-1390.

Fisk, J. E., \& Sharp, C. A. (2004). Age-related impairment in executive functioning: Updating, inhibition, shifting, and access. Journal of Clinical Experimental Neuropsychology, 26(7), 874-890.

Fokkema, T., de Jong Gierveld, J., \& Dykstra, P. A. (2012). Cross-national differences in older adult loneliness. The Journal of Psychology: Interdisciplinary and Applied, 146(1-2), 201-228.

Folstein, M. F., Folstein, S. E., \& McHugh, P. R. (1975). 'Mini-Mental State': A practical method for grading the cognitive state of patients for the clinician. Journal of Psychiatric Research, 12(3), 189-198.
Gow, A. J., Corley, J., Starr, J. M., \& Deary, I. J. (2013). Which social network or support factors are associated with cognitive abilities in old age?. Gerontology, 59(5), 454-463.

Gunzler, D., Chen, T., Wu, P., \& Zhang, H. (2013). Introduction to mediation analysis with structural equation modeling. Shanghai Archives of Psychiatry, 25(6), 390-394.

Hawkley, L. C., Hughes, M. E., Waite, L. J., Masi, C. M., Thisted, R. A., \& Cacioppo, J. T. (2008). From social structure factors to perceptions of relationship quality and loneliness: The Chicago Health, Aging and Social Relations Study. Journals of Gerontology B: Psychological Sciences, 63, 375-384.

Heikkinen, R. L., \& Kauppinen, M. (2004). Depressive symptoms in late life: A 10-year follow-up. Archives of Gerontology and Geriatrics, 38(3), 239-250.

Hu, L., \& Bentler, P. M. (1999). Cutoff criteria for fit indexes in covariance structure analysis: Conventional criteria versus new alternatives. Structural Equation Modeling: A Multidisciplinary Journal, 6(1), $1-55$.

Lai, K., \& Green, G. B. (2016). The problem with having two watches: Assessment of fit when RMSEA and CFI disagree. Multivariate Behavioral Research, 51(2-3), 220-239.

Kenny, R. A., Whelan, B. J., Cronin, H., Kamiya, Y., Kearney, P., O'Regan, C., \& Ziegel, M. (2010). The design of the Irish longitudinal study on ageing. Dublin, Ireland: Trinity College Dublin.

MacKinnon, D. P., \& Fairchild, A. J. (2009). Current directions in mediation analysis. Current Directions in Psychological Science, 18(1), 16-20.

Muyan, M., Chang, E. C., Jilani, Z., Yu, T., Lin, J., \& Hirsch, J. K. (2016). Loneliness and negative affective conditions in adults: Is there any room for hope in predicting anxiety and depressive symptoms? The Journal of Psychology, 150(3), 333-341.

Ofstedal, M. B., Fisher, G. G., \& Herzog, A. R. (2005). Documentation of cognitive functioning measures in the health and retirement study. Institute for Social Research, University of Michigan. Retrieved from http://hrsonline.isr.umich.edu/sitedocs/userg/dr-006.pdf.

Luanaigh, C. O., \& Lawlor, B. A. (2008). Loneliness and the health of older people. International Journal of Geriatric Psychiatry, 23(12), 1213-1221.

Peplau, L. A., \& Perlman, D. (1982). Loneliness: A sourcebook of current theory, research, and therapy New York: Wiley.

Petkus, A. J., Reynolds, C. A., Wetherell, J. L., Kremen, W. S., \& Gatz, M. (2017). Temporal dynamics of cognitive performance and anxiety across older adulthood. Psychology \& Aging, 32(3), 278-292.

Potvin, O., Bergua, V., Meillon, C., Le Goff, M., Bouisson, J., Dartigues, J.-F., \& Amieva, H. (2013). State anxiety and cognitive functioning in older adults. The American Journal of Geriatric Psychiatry, 21(9), 915-924.

Preacher, K. J. (2015). Advances in Mediation Analysis: A survey and synthesis of new developments. Annual Review of Psychology, 66, 825-852.

Qualter, P., Vanhalst, J., Harris, R., Van Roekel, E., Lodder, G., Bangee, M., ... Verhagen, M. (2015). Loneliness across the lifespan. Perspectives on Psychological Science, 10(2), 250-264.

Radloff, L. (1977). The CES-D Scale: A self report depression scale for research in the general population. Applied Psychological Measurement, 1(3), 385-401.

Revelle, W., \& Zinbarg, R. E. (2009). Coefficients alpha, beta, omega and the glb: Comments on Sijtsma. Psychometrika, 74(1), 145-154.

Rigdon, E. E. (1996). CFI versus RMSEA: A comparison of two fit indexes for structural equation modeling. Structural Equation Modeling, 3(4), 369-379.

Rosseel, Y. (2012). lavaan: An R package for structural equation modeling. Journal of Statistical Software, 48(2), 1-36. doi:10.18637/jss. v048.i02

Runge, S. K., Craig, B. M., \& Jim, H. S. (2015). Word recall: Cognitive performance within internet surveys. JMIR Mental Health, 2(2), e20.

Russell, D. W. (1996). UCLA Loneliness Scale (Version 3): Reliability, validity, and factor structure. Journal of Personality Assessment, 66(1), $20-40$.

Saczynski, J. S., Pfeifer, L. A., Masaki, K., Korf, E. S., Laurin, D., White, L., \& Launer, L. J. (2006). The effect of social engagement on incident dementia: The Honolulu-Asia Aging Study. American Journal of Epidemiology, 163(5), 433-440. 
Shao, Z., Janse, E., Visser, K., \& Meyer, A. S. (2014). What do verbal fluency tasks measure? Predictors of verbal fluency performance in older adults. Frontiers in Psychology, 5(1), 772.

Sønderby, L. C., \& Wagoner, B. (2013). Loneliness: An integrative approach. Journal of Integrated Social Sciences, 3(1), 1-29.

Tombaugh, T. N., Kozak, J., \& Rees, L. (1999). Normative data stratified by age and education for two measures of verbal fluency: FAS and animal naming. Archives of Clinical Neuropsychology, 14(2), 167-177.

Weisenbach, S. L., Boore, L. A., \& Kales, H. C. (2012). Depression and cognitive impairment in older adults. Current Psychiatry Reports, 14(4), 280-288.

Wetherell, J. L., Reynolds, C. A., Gatz, M., \& Pedersen, N. L. (2002). Anxiety, cognitive performance, and cognitive decline in normal aging. The Journals of Gerontology Series B: Psychological Sciences and Social Sciences, 57(3), P246-P255.
Wilson, R. S., Krueger, K. R., Arnold, S. E., Schneider, J. A., Kelly, J. F., Barnes, L. L., ... Bennett, D. A. (2007). Loneliness and risk of Alzheimer disease. Archives of General Psychiatry, 64(2), 234-240.

Yaffe, K., Blackwell, T., Gore, R., Sands, L., Reus, V., \& Browner, W. S. (1999). Depressive symptoms and cognitive decline in nondemented elderly women: A prospective study. Archives of General Psychiatry, 56(5), 425-430.

Yochim, B. P., Mueller, A. E., Segal, D. L. (2013). Late life anxiety is associated with decreased memory and executive functioning in community dwelling older adults. Journal of Anxiety Disorders, 27(6), 567-575.

Zigmond, A. S., \& Snaith, R. P. (1983). The hospital anxiety and depression scale. Acta Psychiatrica Scandinavica, 67(6), 361-370. 